HIFAN 1483 LBNL-57320

To be published in Phys. Plasmas

\title{
Simulating electron clouds in heavy-ion accelerators
}

\author{
R. H. Cohen,* A. Friedman, M. Kireeff Covo, S. M, Lund, and A. W. Molvik \\ Lawrence Livermore National Laboratory, Livermore CA 94550 \\ F. M. Bieniosek, P. A. Seidl, and J.-L. Vay \\ Lawrence Berkeley National Laboratory, Berkeley, CA 94720 \\ P. Stoltz and S. Veitzer \\ Tech-X Corporation, Boulder CO
}

(Dated: April 8, 2005)

\begin{abstract}
Contaminating clouds of electrons are a concern for most accelerators of postive-charged particles, but there are some unique aspects of heavy-ion accelerators for fusion and high-energy density physics which make modeling such clouds especially challenging. In particular, self-consistent electron and ion simulation is required, including a particle advance scheme which can follow electrons in regions where electrons are strongly-, weally-, and un-magnetized. We describe our approach to such self-consistency, and in particular a scheme for interpolating between full-orbit (Boris) and drift-kinetic particle pushes that enables electron time steps long compared to the typical gyro period in the magnets. We present tests and applications: simulation of electron clouds produced by three different kinds of sources indicates the sensitivity of the cloud shape to the nature of the source; first-of-e-kind self-consistent simulation of electron-cloud experiments on the High-Current Experiment (HCX) [ P. A. Seidl, D. Baca, F. M. Bieniosek, et al., Proceedings 2003 Particle Accelerator Conference, paper ROAC001 (2003)] at Lawrence Berkeley National Laboratory (LBNL), in which the machine can be flooded with electrons released by impact of the ion beam on an end plate, demonstrate the ability to reproduce key features of the ion-beam phase space; and simulation of a two-stream instability of thin beams in a magnetic field demonstrates the ability of the large-timestep mover to accurately calculate the instability.
\end{abstract}

"Electronic address: rcohenQllnl.gov 


\section{INTRODUCTION}

Heavy-ion accelerators are of interest for their long-term potential application to inertial fusion energy, and for shorter-term applications to high-energy density physics, materials studies, and intense beam physics. Like other postively-charged-particle accelerators, they are subject to contamination by stray electrons, which can be electrostatically trapped by the ion beam potential. This is a phenomenon that has been documented in a range of accelerators dating back to the 1960's [1]; see Refs. [2] and [3] and references therein. The common concern is that the electron cloud is an uncontrolled negative charge that can alter the ion beam dynamics, possibly leading to beam deflection, increased beam emittance, envelope size, and halo, and also potentially electron-ion instabilities. On the other hand, heavy-ion-fusion (HIF) accelerators have a number of distinguishing features that impact both the nature and the modeling of electron clouds.

The distinguishing features of HIF accelerators, along with reports of several simulation studies of electron clouds and electron-cloud effects, were presented in Ref. [4]. In that paper, as well as here, we considered the main-line heavy-ion approach in the U.S., which entails the use of induction linear accelerators, with beam lines having currents of order one to hundreds of Amperes per beam, a system of quadrupole focusing magnets, beam energies ranging from an $\mathrm{MeV}$ to a few $\mathrm{GeV}$ and pulse durations ranging from of order 1 ns to 10 's of $\mu \mathrm{s}$, depending on the application and the part of the accelerator. Ref. [4] noted that the dominant source of electrons in such machines is expected to be ionization of neutral gas desorbed upon beam-ion impact with the beam-pipe wall or direct desorption of electrons, for long or short pulses, respectively. The electron cloud produced by these sources differ: for long-enough pulses, desorbed neutral gas penetrates the beam interior and leads to an electron cloud that is concentrated in the beam interior, whereas, for electrons directly desorbed or born from gas that hasn't had time to move far, the cloud is largely confined by the magnetic field to the pipe edge. For this latter case, it is important to retain the effect of beam-ion scattering at the beam pipe, as that leads to finite (but small, relative to the beam-pipe edge) electron density in the beam interior.

Ref. [4] also describes studies of ion beam propagation in a long (200-quadrupole) system with prescribed (i.e., not self-consistent) model electron cloud distributions. These studies indicate the kinds of electron density perturbations that are likely to have the greatest 
impact, and the perturbation strength required for significant effects. It was found that a constant electron density filling the nominal beam envelope, up to a level as high as $20 \%$ of the beam density, has negligible effect on beam quality (as measured by current, emittance, halo production, and beam envelope evolution) for the system studied. Various types of electron density variations from magnet to magnet were considered; it was found that sinusoidal variations resonant with a natural mode of the ion beam are more effective than random variations, but within each category, amplitude (mean density) variations are more effective than centroid offsets or radial shape variations in producing envelope growth and beam loss. For sinusoidal variations, ellipticity varying resonantly with the beam quadrupole mode was especially effective in increasing the emittance of the beam core, but not in producing envelope growth and beam loss. Finally, Ref. [4] identified an instability associated with amplitude variations resonant with the beam breathing mode and desorption of neutrals at the wall.

The present paper extends that work, describing first results from a self-consistent simulation capability that simultaneously advances electrons, ions, and the electrostatic fields they generate in an accelerator setting that includes magnet regions (where electrons are strongly magnetized) and gaps (where there is no magnetic field). The simulations, done with the WARP particle-in-cell (PIC) code[5], also include the important effects of electron desorption caused by ion impact and secondary electron production. The paper also describes experiments dedicated to electron-cloud effects in the High-Current Experiment (HCX)[6] at Lawrence Berkeley National Laboratory, and comparisons of theory and experiment.

The remainder of this paper is organized as follows. In Sec. II, we summarize the key ingredients of our current simulation model, including our new long-timestep electron mover that enables stepping of electrons on a timescale governed by the electron bounce time in the electrostatic potential well, independent of the strength of the magnetic field. A demanding test of the mover in a textbook-like context, calculation of the growth of twostream instability of thin beams in a. uniform magnetic field, is described in the Appendix. Other tests of the mover appear within the context of the applications described in the remaining sections. Section III describes experiments dedicated to electron-cloud effects in the High-Current Experiment (HCX)[6] at Lawrence Berkeley National Laboratory, and our simulation of these experiments. Section $\mathrm{TV}$ is a comparison of the electron clouds produced by three different types of electron sources - direct electron desorption at end plates, a 
volumetric source such as is obtained by fonization of neutral gas filing the beam pipe, and electron desorption from ion beam scrape-off at the beam pipe. Section V is a summary and discussion of the results.

\section{SIMULATION MODEL}

Modeling electron cloud effects in heavy-ion accelerators (and, we believe, other accelerators as well) requires self-consistent solution of electrons and ions. This is because the dominant sources of electrons are associated with loss of beam ions, and (as shown in Ref. [4]) the interaction of electrons with ions alters the ion beam propagation in such a way as to alter ion beam loss. Furthermore, the electron dynamics depends on the ion distribution becaue of its high space charge potential. Hence, a one-way chain of calculations is insufficient.

Our approach to self-consistent electron and ion simulation has been to extend the WARP code. WARP at its core is a multi-species three-dimensional electrostatic particle-in-cell (PIC) code, with specialized capabilities to include the applied magnetic and electrostatic fields and bounding conductors found in particle accelerators. To this core, we have added modules for secondary electron emission and ion-induced electron desorption [from the Computational Modules for Electron Effects (CMEE) library[7], derived from routines in the POSINST high-energy-physics accelerator code[8]), first-cut models for ion reflection at walls and ionization source terms, and the large-timestep electron mover described below. We have, in development off-line, models for neutral-gas desorption and transport, charge exchange, and improved models of ion reflection and ionization.

Self-consistent simulation of electrons and ions requires simulation of electrons in the quadrupole magnets as well as in the gaps between magnets, and running the simulation long enough to simulate the passage of the ion beam. This results in a broad range of time scales, ranging from the electron cyclotron period $\left(10^{-10}-10^{-11} \mathrm{~s}\right)$ through the ion beam transit time $\left(10^{-5}-10^{-7} \mathrm{~s}\right)$. The shortest electron cyclotron period is typically one to two orders of magnitude shorter than the next-shortest timescale, usually the electron bounce time in the combined beam-potential and magnetic wells.

We have developed a mover for electrons that interpolates between full electron dynamics and drift linetics. The algorithm is briefly mentioned in Ref. [4]. The algorithm builds upon 
the observation by Parker and Birdsall [9] that the conventional Boris particle advance scheme, when run with time steps large compared to the cyclotron period, continues to exhibit correct drift velocities, but causes particles to gyrate with a radius that is large compared to the physical gyro orbit, and with a frequency that is lower than the physical gyrofrequency. Our interpolation scheme corrects the former deficiency, preserving a physical gyroradius, and is thus well suited for simulating particles that move through regions of strong, weak, and no magnetic field such as we have in HIF accelerators.

Specifically, we interpolate in the velocity perpendicular to the magnetic field. Schematically, we proceed to advance the velocity in a conventional manner,

$$
\mathbf{v}_{\text {new }}=\mathbf{v}_{\text {old }}+\Delta t\left[\left(\frac{d \mathbf{v}}{d t}\right)_{\text {Lorentz }}+(1-\alpha)\left(\frac{d \mathbf{v}}{d t}\right)_{\mu \nabla B}\right]
$$

and then advance the particle position using an effective velocity which is an interpolation of this updated velocity and the drift velocity:

$$
\mathbf{v}_{e f f}=\mathbf{b}(\mathbf{b} \cdot \mathbf{v})+\alpha \mathbf{v}_{\perp}+(1-\alpha) \mathbf{v}_{d}
$$

Here, the first equation denotes an update of the velocity under the combined influence of electric and magnetic fields (Lorentz force) as in the standard Boris algorithm[10], to which is added a rotation of the velocity in the plane of $\mathbf{v}$ and $\mathbf{B}$ such as to effect the $\mu \nabla B$ acceleration of the parallel velocity that is needed in drift linetics ( $\mu$ is the magnetic moment). In the second equation, $\mathbf{v}_{d}$ denotes the drift velocity (sum of electric and magnetic drifts), $\alpha$ is an interpolation parameter, and $\mathbf{b}=\mathbf{B} / B$.

For the particular choice of interpolation parameter $\alpha=1 /\left[1+\left(\omega_{c} \delta t / 2\right)^{2}\right]^{1 / 2}$, the radius of the gyration motion is physically correct for large as well as small $\omega_{c} \delta t$ The drift is physically

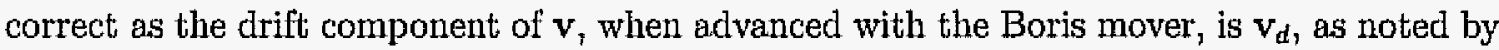
Parker and Birdsall[9]. And, finally, the parallel dynamics is correct as the full particle push in the direction of the magnetic field is retained along with the $\mu \nabla B$ correction. However, it should be noted that accurate results require attention to a number of details (e.g. centering) which cannot be discussed here; these will be spelled out in a separate paper devoted to the algorithm.

The mover has been tested extensively with respect to single-particle dynamics; we find that it agrees well with small-timestep solutions for drift and bounce velocities and gyration radius, and also exhibits a transition from adiabatic (conserving magnetic-moment) 
to nonadiabatic (large jumps in magnetic moment) behavior at about the correct value of the particle energy (or equivalently, at about the correct distance of closest approach to the center of a quadrupole magnetic field). These tests will be discussed in the algorithm paper. We have also performed a number of tests involving large ensembles of test particles and fully self-consistent simulations. One of the former category, calculation of the electron cloud distribution resulting from desorption upon ion wall impact, was described in Ref. [4]. Several other tests are described in the remaining sections of this paper.

\section{SIMULATION OF HCX ELECTRON-CLOUD EXPERIMENTS}

A series of experiments dedicated to production and measurement of the effects of electron clouds has been carried out on the High-Current Experiment (HCX), and simulated with WARP. The electron studies are performed in four quadrupole magnets (MA1-4) on the High Current Experiment (HCX) facility, as shown in Fig. 1. These magnets follow $10 \mathrm{HCX}$ electrostatic quadrupoles through which the $1 \mathrm{MeV}, 174 \mathrm{~mA} \mathrm{~K} \mathrm{~K}^{+}$ion beam was transported with little or no degradation [11].

In the experiments, the ion beam is allowed to impact a plate downstream of the last quadrupole magnet. This should result in emission of a copious supply of electrons. This conclusion is based on extrapolation to normal incidence of measurements of electron emission from $1 \mathrm{MeV} \mathrm{K}^{+}$ions impinging upon a stainless plate near grazing incidence [12], implying an electron emission coefficient of 6. (This coefficent agrees with theoretical estimates [7], although other HIF experiments have suggested that electron emission coefficients can be as high as 10-30). A suppressor ring electrode is mounted between the final magnet and the end plate. This electrode can be biased to $-10 \mathrm{kV}$ to repel back the electrons emitted from the plate, or it can be left grounded to allow electrons to propagate upstream. There is also a series of three clearing electrodes (see Fig. 1) in the drift regions between quadrupole magnets, which can be biased to draw off electrons from between any pair of magnets. The current under these bias conditions provides a measure of the flux of electrons traversing the magnets downstream of the last biased electrode. The end plate itself is movable and contains a slit (there are actually two such platies, 26 and $28.5 \mathrm{~cm}$ downstream from the fourth magnet, with the slits oriented vertically and horizontally, respectively); scintillatior images obteined further downstream provide information about slices of the beam phase 
space. By combining the images from different slit positions, one can reconstruct the $x-x^{\prime}$ or $y-y^{\prime}$ phase space of the ion beam at, the plate. (Here' denotes $d / d z$, where $\mathrm{z}$ is in the ion beam propagation direction.)

Data were taken with various combinations of biased and unbiased suppressor and clearing electrodes. In particular, there is a striling contrast in the $x-x^{\prime}$ phase space between the case where the suppressor is on (no electrons from the slit plate penetrate upstream) and when the suppressor and all clearing electrodes are unbiased. These are shown, respectively, in Figs. 2 and 3. In particular, with the first clearing electrode (" $\mathrm{a}$ ") biased, and all remaining electrodes, including the suppressor ring, unbiased, there is a distinct " $z$ " character (that is, $x^{\prime}$ increasing less than linearly, or decreasing, with increasing $x$ ) to the phase space, whereas there is a bit of "z-ing" but much less for the case where the suppressor electrode is biased. This " $\mathrm{z}$ " character represents a significant departure from the linear relationship between $x$ and $x^{\prime}$ that would result from perfect linear focussing $g_{1}$ and so is indicative of a significant degradation in beam quality. There is little difference in the slit images for any combination of clearing electrode biases when the suppressor ring is biased. It should be noted that there is some "z-ing" even with the suppressor on, whereas there is none upstream of the magnetic quadrupoles. This is suggestive of a residual population of electrons even in the absence of the slit plate source.

By examining the current in the (negatively) biased clearing electrodes, inferences can be made about the electron density.(No significant current, is drawn by an unbiased electrode.) The currents with the suppressor off are 2 to 4 times higher than with the suppressor on, indicating that flow from the end plate is indeed a significant source of electrons, but, probably, not the only source; for example, ionization of background and desorbed neutral gas is a likely additional source. With the suppressor off, the current to the downstream-most, biased electrode is - within a factor of two - independent of which of the three elctrodes has that role, and uniformly higher (by 2 to 4 ) than with the suppressor on. This suggests that electrons that survive to exit upstream from the fourth magnet have a significant probability of finding their way through the remaining magnets if the intervening clearing electrodes are unbiased. And, comparing the current to the last biased electrode to the beam current, along with inferences about the electron drift velocity and effective cross sectional area from the simulation results described below, suggests that the electron density is comparable to the beam density when the suppressor is off. 
To simulate these experiments, the experimental setup described in Fig. I was reproduced in a WARP input file. Quadrupole fields are represented by a high-order multipole expansion while conductors (beam pipes and diagnostic plates) are embedded in the Poisson solver using the cut-cell method $[13,14]$. The computational zone extends longitudinally from the exit plane of the last electrostatic quadrupole to the slit plate, located $26 \mathrm{~cm}$ downstream of the last magnetic quadrupole exit. Transversely, it extends from the beamline axis to 10 $\mathrm{cm}$ both in $\mathrm{X}$ and in $\mathrm{Y}$. Fourfold symmetry was assumed (and a single quadrant simulated) to reduce the computation time. The simulation includes a representation of the elliptical beam pipe (alternating axes in successive magnets) as a grounded surface. The transverse domain size was chosen to be approximately twice the major diameter of the beam pipe in order to allow for possible large transverse excursions of electrons in the regions between the magnets and between the last magnet and the diagnostic plate, where there is no beam pipe. The beam was launched at the exit of the last electrostatic quadrupole using the timehistories of the beam current, energy, transverse edge envelope dimensions and velocities, and emittances, all derived from experimental data. The initial phase-space structure of the beam was not taken from detailed experimental data; instead, a fitted semi-Gaussian profile (flat in coordinate space, Gaussian in velocity space) was assumed. Beam ion macroparticles reaching the slit plate generated 6 macroelectrons each, in accord with the discussion above. A temperature of the emitted electrons of $10 \mathrm{eV}$ was assumed in the results shown; runs with different initial temperatures (up to $30 \mathrm{eV}$ ) indicate little sensitivity to this value.

Electrons and ions are followed simultaneously, with a timestep chosen to adequately resolve the electron bounce motion in the magnetic and beam potential wells. The choice, $\Delta t=10^{-10} \mathrm{sec}$, corresponds to about a cyclotron period near the transverse edge of the resultant electron cloud in the quadrupole magnets. When an electron hits a conducting surface, it (depending on the run) is either absorbed, or produces secondary electrons in accord with the CMEE secondary-electron model mentioned in Sec. II. At this time the reutral gas modules are not yet operational in the code; hence we cannot yet simulate what may be important local sources of electrons.

Results for the $x-x^{\prime}$ ion phase space are shown for a case with no electrons (Fig, 4), and with all electrodes unbiased and with secondary electron emission (Fig. 5). It is seen that strong "z-ing" has developed by the end of the simulation run ( $4 \mu \mathrm{s}$, as in the experiment) with electrons in all quads, but very little nonlinearity of any lind develops with no electrons. 
A run with unbiased electrodes and no secondary electrons looks very similar to Fig. 5).

Results for the electron distribution without, and with, secondary emission are shown in Figs. 6 and 7 , respectively. It can be seen that there are significant differences in the electron distributions; in particular, the electron density is significantly greater in the upstream magnets when secondary electrons are included, and the electron density in the fourth magnet is more symmetric with respect to the quadrant distribution. These phenomena are both attributable to the presence of a significant flux of electrons to the beam pipe just inside the entrance of the fourth magnet, which in turn results from the turning points of electrons tracking equipotential surfaces as they drift upstream. This flux constitutes a sink of electrons in the absence of secondary emission. The inclusion of secondaries thus allows the simulation to obtain electron fluxes and densities that extend more through the upstream magnets, in closer agreement with the experimental inferences disctussed above. In either case the electron density in the fourth magnet is approximately equal to the beam density, again consistent with that inferred from the experiment.

\section{DEPENDENCE OF ELECTRON CLOUD SHAPE ON ELECTRON SOURCE TYPE}

We describe in this section several calculations which serve both to test the large-timestep electron mover and also to elucidate the dependence of the electron cloud distribution on the nature of the electron source.

The first case we consider is a restricted version of the self-consistent HCX simulation described in the preceding section. Here we consider only the final (fourth) magnet and the end region, to study the shape of the electron cloud produced by desorption upon ion bombardment of the slit plate. In this simulation, secondary emission is turned off, and electrons which emerge up-stream of the fourth magnet are reflected at what would be the entrance to the third magnet. Electrons can only enter the fourth magnet from down (up) stream in two of the 4 quadrants, namely those for which the electric and magnetic drifts point up- (down-) stream. The results are shown in Fig. 8 using the interpolated mover with the same timestep as in the last section (time step $\delta t \sim$ cyclotron period $\tau_{B}$ ), and also for two other cases: a factor of ten smaller timestep, and the larger timestep but with a straight Boris particle push (the scheme of Parker and Birdsall[9]). We notice that the 
interpolated and small-timestep results agree very well, but there are significant differences when compared to the large-timestep Boris/Parker-Birdsall result. (The same conclusions apply to the transverse distributions, though only the large-timestep, interpolated result is shown here). This comparison gives confidence in the results shown in the preceding section. The presence of electrons in predominantly two of the four quadrants of the quadrupole magnet is the result of the fact that there is a significant sink of electrons at the pipe wall. Hence the reservoir of electrons in the gap upstream of the 4th magnet is less filled than that in the end tank, thus accounting for the asymmetry.

The second case is loading of low-temperature $(10 \mathrm{eV})$ electrons in a quadrupole magnet, uniformly out to a radius equal to the nominal (mean) ion beam radius. This is representative of what one might expect from ionization of neutral gas that fills the beam pipe (either ambient, or from wall desorption if there is sufficient time for the neutrals to propagate). The example is artificial in the sense that the electrons are loaded at the start of the run rather than continuously, and is strictly a electron test-particle simulation; the ions are represented as a fixed positive charge filling the beam envelope (as computed from envelope equations), and the only electric field used is the one calculated for the fixed positive charge. The resultant electron distribution is not steady during the course of the run; what is shown in Fig. 9 is a snapshot in time, again for $\delta t \sim \tau_{B}$ with the interpolated mover and standard Boris mover, and for $\delta t$ ten times smaller).

As a final case, we display the results of the study of Ref. [4], which computes the electron cloud resulting from direct electron desorption associated with computed loss of primary and scattered beam ions at the radial wall. A time-averaged $x-y$ density plot, integrated over the length of the multi-magnet system, is shown in Fig. 10. We show here only the largetimestep interpolated-mover result; excellent agreement with a simulation which resolved the cyclotron period ( 25 times smaller timestep) was shown in Ref. [4].

Comparing the $x-y$ plots for the three different electron soruces, we see significant differences, which are readily understood in terms of the nature of the sources. Electrons borr at the end wall enter the quadrupole magnets with energies comparable to the beam space charge potential, and, thanks to the action of the fringe magnetic field at the magnet entrance, have a broad distribution of pitch angle (the angle between the velocity vector and the magnetic field). Hence electrons which enter the magnet within the footprint of the ion beam can follow field lines well beyond the footprint of the beam before they turn 
around. As they do so they concentrate around the principal diagonals ( 45 and 135 degrees in the $x-y$ plane), as this is what the quadrupole field lines do. In contrast, the low-energy electrons in the second study are well confined by the beam potential. Those born near the principal diagonals are accelerated by it and their density decreases; those born mid-way between cannot gain significant energy from the beam potential and hence their density remains relatively high. These observations account for the relatively low density near the principal diagonals, and the overall radial confinement. Finally, electrons desorbed from the wall are, except for electrons born close to the principal diagonals, confined close to the wall by the magnetic field. This is especially true for electrons born from primary ion beam impact, which occurs primarily at the vertical and horizontal axes, as noted in Ref. [4].

\section{DISCUSSION}

We have presented simulation results for simultaneous electron and ion simulation in a beam transport system containing both quadrupole magnetic fields and magnetic-fieldfree regions. These results, which we believe are first-of-a-kind, represent a snapshot of an evolving capability to self-consistently model electron clouds in ion-beam acceleratiors and transport sytems. In particular, a number of enhancements in development must be operational before the capability will be complete. This includes models for gas desorption and transport and volumetric ionization, and an improved model for ion reflection at bounding surfaces. The completed package will be a valuable tool for simulation of electron clouds in a variety of accelerators.

The WARP simulations of the electron-cloud experiments on HCX have encouraging results (qualitatively similar phase-space distortions and overall electron density level), but are presently limited by the missing simulation ingredients noted above. Another limitation is the treatment of injected electrons at the end plate: the present model, which does not resolve the sheath region, may miss important aspects of interaction of the electron cloud with the electrostatic potential near the plate. This in turn can affect the electron speed distribution in the quadrupole magnet, and produce errors in the flux lost to the radial wall. We will address this issue in future runs by exercising WARP's mesh refinement capabilities.

We have presented a number of examples of application of the large-timestep interpolated mover, which indicate that the mover works quite well, reproducing the results from small- 
timestep simulations and in particular (as the example in the Appendix shows) properly capturing finite-gyroradius effects. Use of the mover allows the simulation to proceed on the next-shortest time scale, which is the electron bounce time, typically one to two orders of magnitude larger than the shortest cyclotron period. This is a significant advance, and for some types of simulations it is the best one can hope for - for example, it is needed to follow the evolution of an electron-ion two-stream instability[15] in a long system, as the electron bounce time is of physical interest. For other problems, one would like to be able to simulate on ion-transit timescales, which are typically (though not always) another order of magnitude larger. Options to consider include electron sub-cycling, bounce averaging, and projective integration techniques.

Finally we comment on the longitudinal electron-density striations observed in the HCX simulations (see the $y-z$ plots in Figs. 6, 7, and 8). These patterns are observed for large and small time steps, with and without secondary emission, and are observed, from examination of plots at different times, to propagate. While we have not completed a formal analysis, it is clear that there is a mechanism for a drift instability associated with a perturbation in density of an opposite-charge minority species for a beam in a magnetic field. Consider a region with a localized electron density enhancement (but still the net line-charge density is positive). In this region, the net space charge is reduced, and hence the $E x B$ drift velocity is reduced. This acts to increase the density perturbation. Similarly, if the beam resides inside a grounded beam pipe, the equipotential surfaces bow inward in regions where the electron density is enhanced, compressing electron bounce orbits there and so further increasing the density. The propagation of these density striations could account for current fluctuations observed in the last biased clearing electrode (and observed only when the suppressor electrode is unbiased).

\section{Acknowledgments}

We thank D. P. Grote for invaluable assistance with the WARP code, M. A. Furman and J. Verboncoeur for useful discussions, and C. Celata for many comments on the manuscript. This work was performed under the auspices of the U.S. Department of Energy by Lawrence Livermore National Laboratory under contract W7405-ENG-48 and by Lawrence Berkeley National Laboratory under contract DE-AC03-76F00098, and under an SBIR grant at Tech- 
X.

\section{APPENDIX A: TWO-STREAM INSTABILITY}

We consider a test problem umrelated to electron clouds in accelerators, but which serves to illustrate the broader potential applicability of our large-timestep algorithm. The problem is that of two-stream instability of thin counterstreaming beams. This problem is specified so that it is simple to compute and yet exhibits the value of the algorithm, including its ability to capture physically correct finite-gyroradius effects.

We consider infinitely long ion beams counterstreaming along a uniform magnetic field $\mathbf{B}$ (1 T), with a finite temperature perpendicular to $\mathbf{B}$ and a much (1000 times) smaller parallel temperature. We specify the gyroradius $(1.5 \mathrm{~cm})$ and a beam radius $r_{b}$ which is 10 times the gyroradius, and load guiding center positions uniformly out to the beam radius. The speed of the beams $v_{b}$ is taken to be 0.1 times the perpendicular thermal speed, and the simulation volume is taken as a cylinder, 4 times the nominal beam radius across, and with a length $4 \pi v_{b} / \omega_{p}$ where $\omega_{p}$ is the plasma frequency (periodic boundary conditions in $z$ ). We take the cyclotron frequency to be large compared to the plasma frequency, $\omega_{c} / \omega_{p}=48$. Since this is also the ratio of the Debye length $\Lambda_{D}$ to the gyroradius, we notice that our beam is only about $1 / 5$ of a Debye length in radius, and the system is about one wavelength long. The simulations are done in two-dimensional cylindrical geometry with periodic boundary conditions in the axial coordinate $z$.

This is not a "textbook" two-stream problem, because of the small beam radius. However, it does exhibit two-stream instability, though the strength of the instability is reduced as $r_{b} / \Lambda_{D}$ is decreased, and it is from this that our "finite gyroradius" effect arises: the effective perpendicular Debye length scales with gyroradius (at fixed $B$ and beam density).

The results of the test are shown in Fig. 11. There we compare the growth of the potential perturbation, measured at a single point in space, for the problem as specified, with small timesteps $\left(\omega_{c} \delta t=0.25\right)$, with large $\left(\omega_{c} \delta t=5\right)$ timesteps integrated with the interpolated mover described in Sec. II, and with the same large timesteps integrated with a pure Boris mover (Parker-Birdsall scheme). Also for comparison we show the calculation (with the long-timestep interpolated mover) for a beam that is twice as large. Comparing results, we see that the interpolated mover reproduces very well, over two decades, the instability 
growth, saturation, and even the subsequent nonlinear evolution. The primary difference is a small time shift in the curves, which can be attributed to the difference in random seed from particle noise (the particle positions differ for any time after the start of the run, because of the reduced effective gyration frequency of the interpolated mover). In contrast, the large-timestep run with the pure Boris mover does not develop two-stream instability, while the reference case with twice the beam radius exhibits a larger growth. The $z$ phaseplane plots for the small-timestep and interpolated-mover runs are very similar as well; Fig. 12 shows such plots near the peak of the potential growth curve. The primary differences in the figures shown are due to the rather course sampling of the simulation to produce scatter plots (plots were not made at just the same level of instability growth). There is also a small shift in phase $(z)$ between the two plots ${ }_{\uparrow}^{+}$this is not surprising since the phase depends on the shot-noise seed. The position where the potential history is plotted is shifted for the interpolated mover (Fig. 11b), to account for this phase shift.

Not shown are the $x-y$ scatter plots; for both the small-timestep and interpolated runs, these plots show a disk that is of the same size as the originally loaded distribution. In contrast, for the pure Boris large-timestep simulation, the beam radius (after an initial transient) slowly oscillates between about two and four times the initial beam radius. At this point we do not understand these oscillations - they are too big in amplitude (by about a factor of two) to be simply the anomalous "gyro" oscillations noted by Parker and Birdsall[9], and are also too slow. However, given the existence of these oscillations, they could explain the absence of two-stream instability in that simulation; the continually evolving plasma frequency could thwart growth of instability. 
[1] G. Budker, G. Dimov, and V. Dudnikov, Sov. Atom. E. 22, 5 (1967)

[2] Proc. Workshop on Electron-Cloud Simulations for Proton and Positron Beams (ECLOUD 02), CERN, Geneva, April 15-18, 2002, CERN Report CERN-2002-001 (CERN, Geneva, 2002), ISBN 92-9083-193-6 http:// wwwslap.cern.ch/collective/ecloud02/proceedings

[3] Proc. 31st ICFA Advanced Beam Dynamics Workshop on Electron-Cloud Effects (ECLOUD'04), Napa, CA, USA, 19-23 Apr 2004, CERN Report CERN-2005-001 (2005), ISBN 92-9083-241-X, http://icfa-ecloud04.web.cern.ch/icfa-ecloud04/agenda.html

[4] R. H. Cohen, A. Friedman, S. M. Lund, A. W. Molvik, E. P. Lee, T. Azevedo, J.-L. Vay, P. Stoltz, S. Veitzer, "Electron-Cloud Simulation and Theory for High-Current Heavy-Ion Beams," Phys. Rev. Special Topics-Accelerators and Beams 7, 124201 (2004).

[5] D. P. Grote, A. Friedman, I. Haber, Fus. Eng. \& Des., 32-33, 193 (1996) and http://hif.lbl.gov/theory/WARP_summary.html

[6] P. A. Seidl, D. Baca, F. M. Bieniosels, C. M. Celata, A. Faltens, L. R. Prost, G. Sabbi, W. L. Waldron, R. Cohen, A. Friedman, 5. M. Lund, A. W. Molvik, Proceedings 2003 Particle Accelerator Conference, paper ROAC001 (IEEE, Piscataway, NJ, 2003); http://accelconf.web.cern.ch/AccelConf/p03/PAPERS/ROAC001.pdf

[7] P. H. Stoltz, M. A. Furman, J. L. Vay, A. W. Molvik and R. H. Gohen, Phys. Rev. ST Accel. Beams 6, 054701 (2003)

[8] M. T. F. Pivi and M. A. Furman, Phys.Rev. ST Accel. Beams, 6, 034201 (2003).

[9] S. E. Parker and C. K. Birdsall,J. Comp. Phys 97, 91 (1991).

[10] J. P. Boris, "Relativistic Plasma Simulation - Optimization of a Hybrid Code," in Proc. 4th International Conference on Numerical Simulation of Plasmas, Washington, DC, 2-3 November, 1970 (Naval Research Lab, Washington, 1970; U.S. Government Printing Office, Stock Number 085100059 ), p. 3.

[11] L. Prost, D. Baca, F. M. Bieniosek, C. M. Celata, A. Faltens, E. Henestroza, J. W. Kwan, M. Leitner, P. A. Seidl, W. L. Waldron, R. Cohen, A. Friedman, D. Grote, S. M. Lund, A. W. Molvik and E. Morse, submitted to Phys. Rev. Special Topics-Accelerators and Beams (2004).

[12] A. W. Molvik, M. Kireeff Covo, F. M. Bieniosek, L. Prost, P. A. Seidl, D. Baca, A. Coorey, 
and A. Sakumi, Plyss. Rev. Special Topics-Accelerators and Beams 7, 093202 (2004).

[13] G. H. Shortley and R. Weller, J. Applied Physics, 9, 334 (1938).

[14] D. P. Grote, A. Friedman, I. Haber and S. Yu, Fusion Engineering and Desígn 32-33, 193 (1996).

[1.5] R. C. Davidson, H. Qin, P. H. Stoltz, T-S. F. Wang Phys. Rev. ST-Accel. Beams 2, 054401 (1999) 


\section{Figure Captions}

FIG. 1: Electron studies are performed in 4 quadrupole magnets (MA1-MA4) with elliptical bores on the HCX. A suppressor electrode is shown at the right; the electrodes (a) - (c) are clearing electrodes in the drift regions between quadrupole magnets. Every other magnet is rotated $90^{\circ}$, as indicated by the alternating ( 6 and $10 \mathrm{~cm}$ ) diameters of the shown beam pipe cross section. A diagnostics region is to the left of MAI.

FIG. 2: Reconstruction of $x-x^{\prime}$ phase space from scan of slit data, with suppressor on and clearing electrodes off. (File 409220259-296xx)

FIG. 3: Reconstruction of $x-x^{\prime}$ phase space from scan of slit data, with suppressor off and only the first clearing electrode biased, at $+9 \mathrm{kV}$. (File 409220222-257xx)

FIG. 4: Ion horizontal $\left(x-x^{\prime}\right)$ phase space at end plate for WARP HCX simulation with no electrons

FIG. 5: Ion horizontal phase space at end plate for WARP HCX simulation with electrons

FIG. 6: Electron spatial distribution in (a)transverse $(x-y)$ in fourth magnet and (b) vertical $(y-z)$ planes for simulation without secondary electrons. Ion distribution (black) is shown under electrons

FIG. 7: Electron spatial distribution in (a)transverse $(x-y)$ and (b) vertical $(y-z)$ planes for simulation including secondary electron emission

FIG. 8: Instantaneous electron spatial distribution in vertical $(y-z)$ plane for 4th-magnet-only HCX simulation: (a) using small timesteps; (b) with large timesteps and the interpolated mover; (c) with large timesteps and the standard Boris mover; (d) is transverse ( $x-y$ ) distribution (result shown is from interpolated mover, and looks very similar to result using small timesteps). Ion distribution is shown in black underneath electrons. 
FIG. 9: Instantaneous electron spatial distribution in transverse $(x-y)$ plane for test-particle simulation of low-temperature electrons injected uniformily within a cylindrical slice: (a) using small timesteps; (b) with large timesteps and the interpolated mover; (c) with large timesteps and the standard Boris mover

FIG. 10: Averaged electron spatial distribution in transverse $(x-y)$ plane for test-particle simulation of wall-desorbed electrons

FIG. 11: Potential versus time for pencil-beam two-stream instability (a) using small timesteps; (b) with large timesteps and the interpolated mover; (c) with large timesteps and the standard Boris mover; (d) for a beam twice the size (with large timesteps and interpolated mover). Potential is measured at the center of the cylinrical simulation volume for (a) and (c) and shifted for (b) to account for the phase shift seen in Fig. 12.

FIG. 12: Longitudinal $\left(z-v_{z}\right)$ phase space distribution at times near the peak of the potential (a) using small timesteps; and (b) with large timesteps and the interpolated mover. 


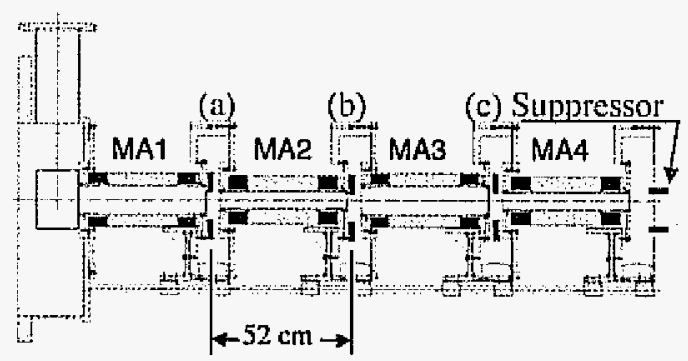

Figure 1 


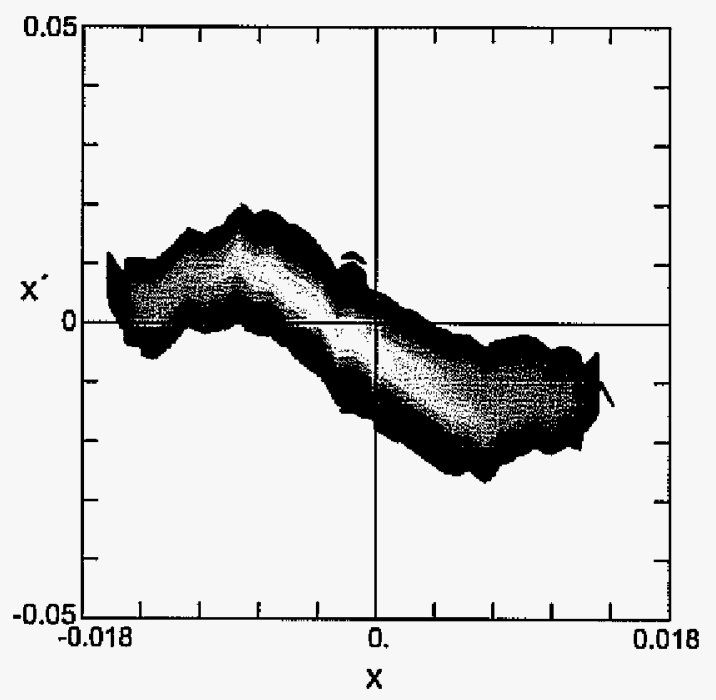

Figure 2 


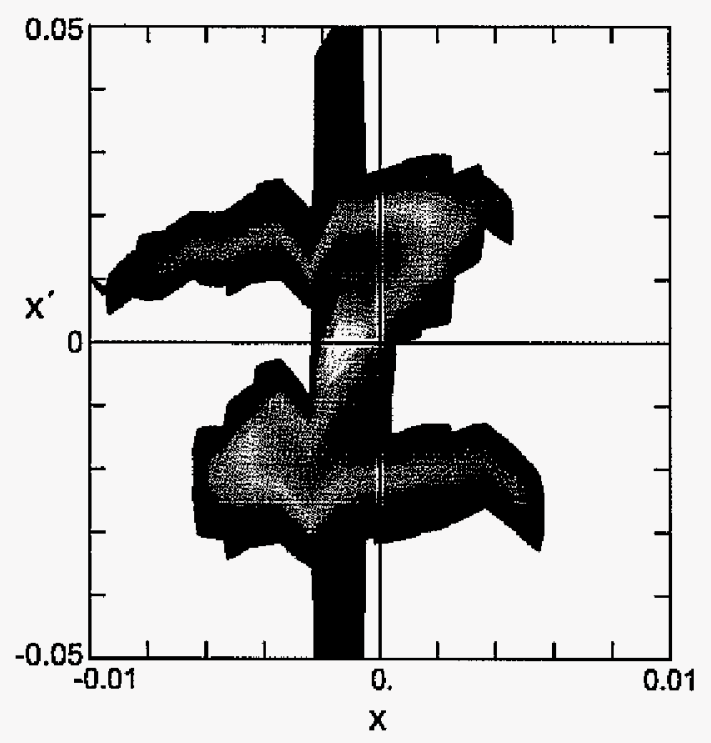

Figure 3 


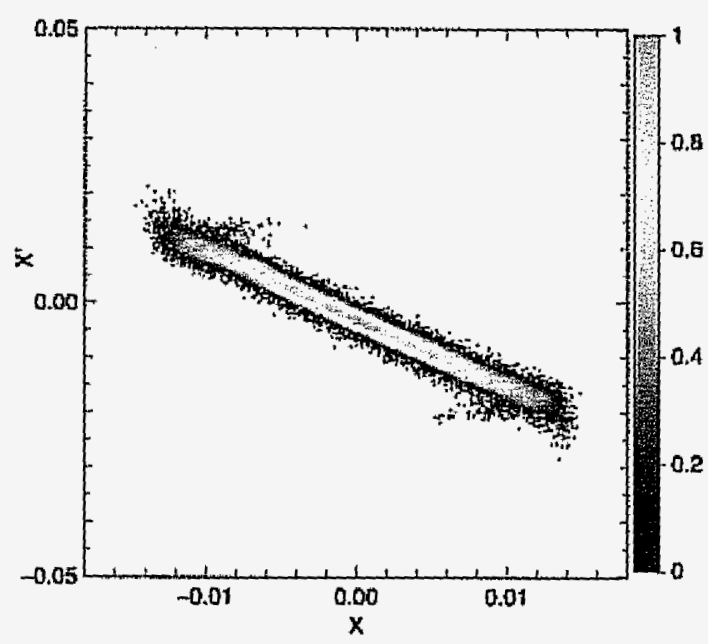

Figure 4 


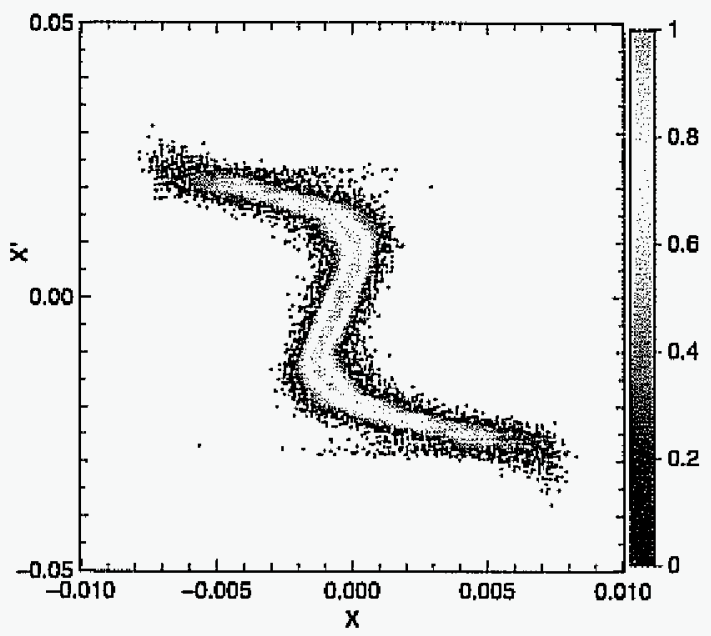

Figure 5 

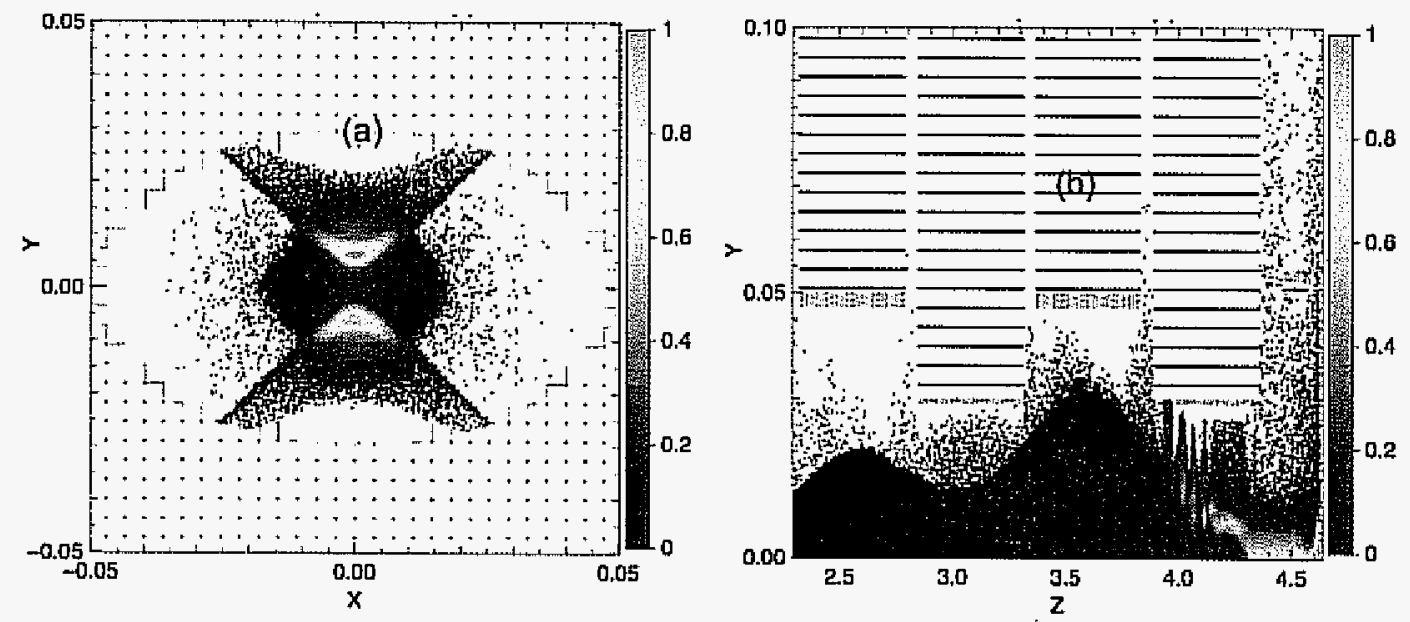

Figure 6 


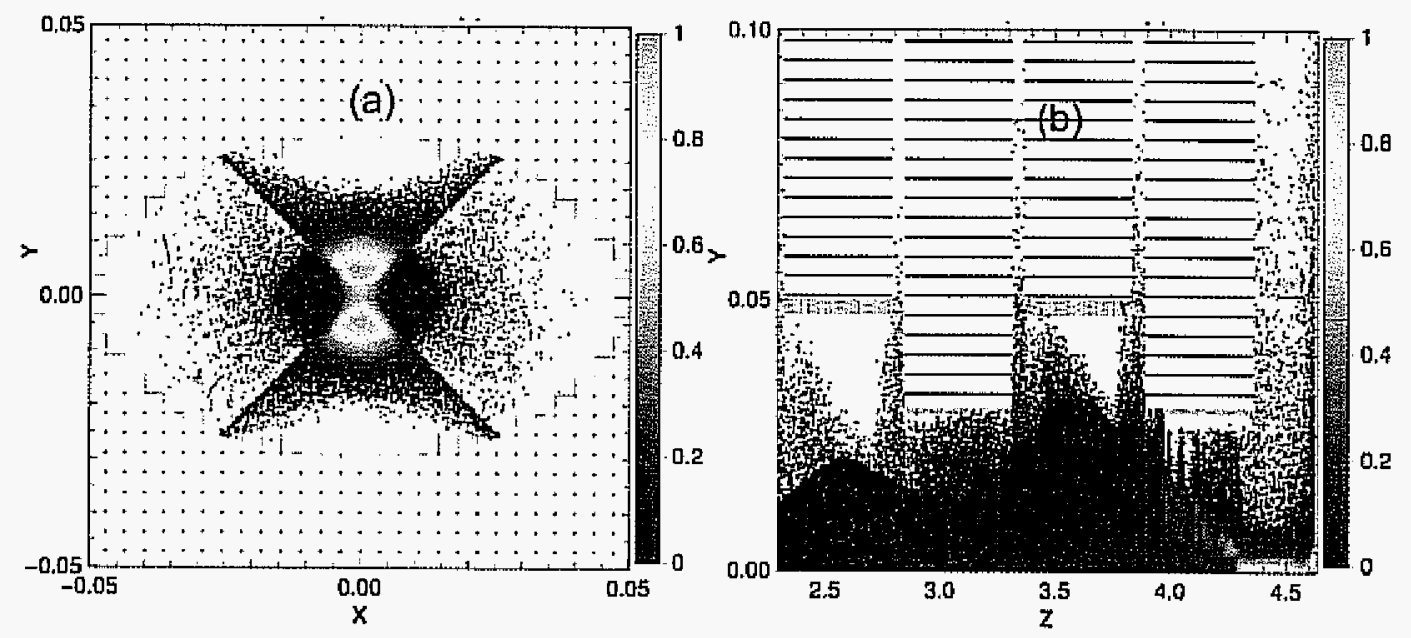

Figure 7 


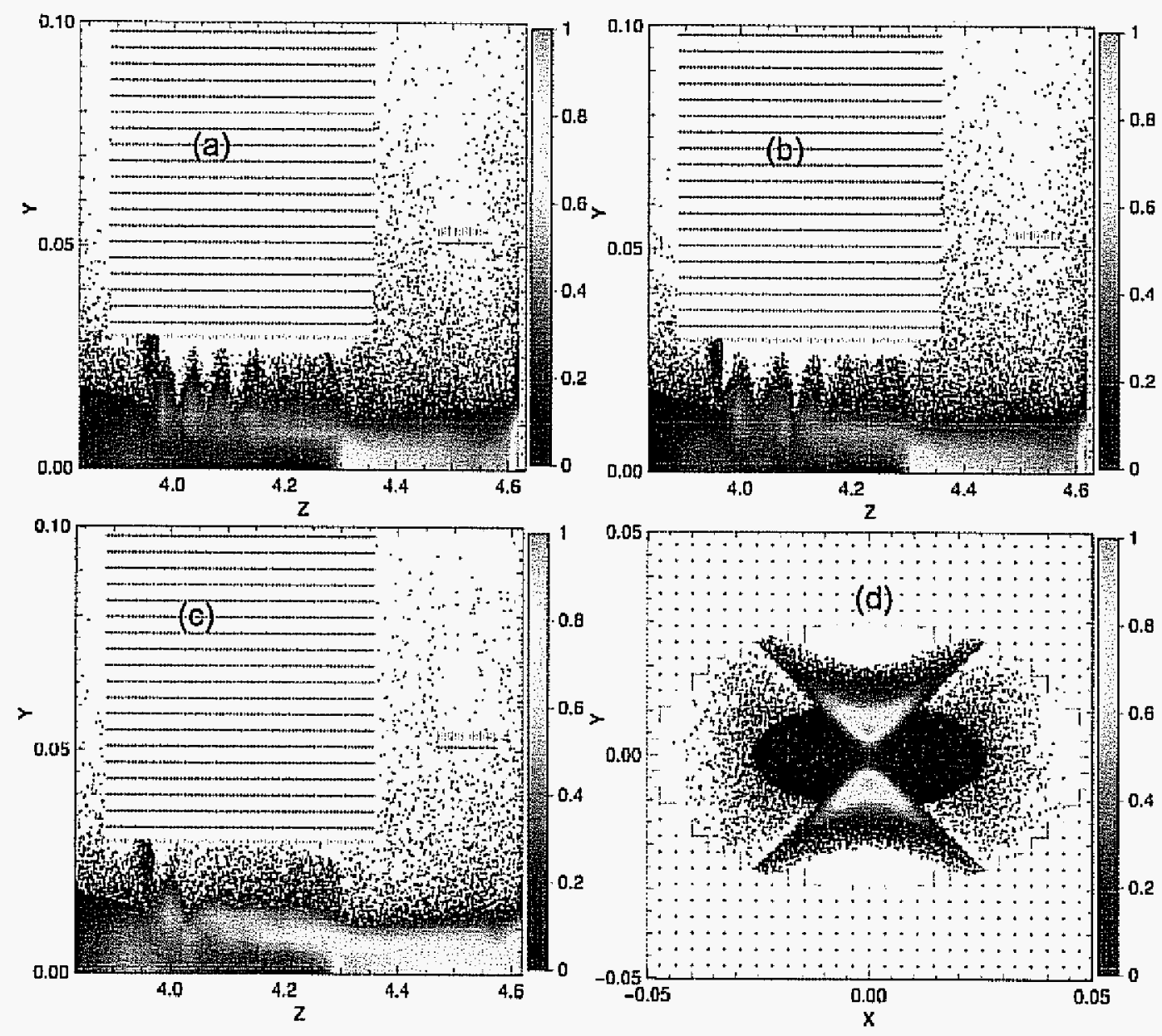

Figure 8 

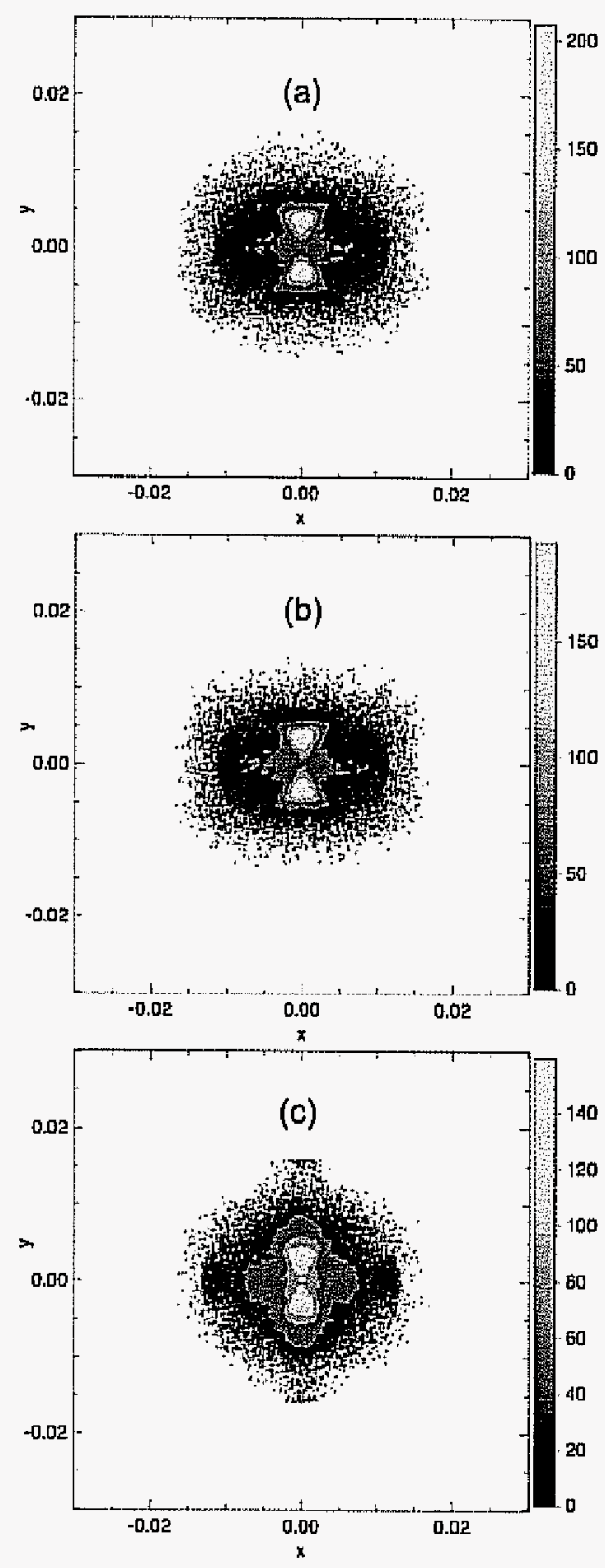

Figure 9 


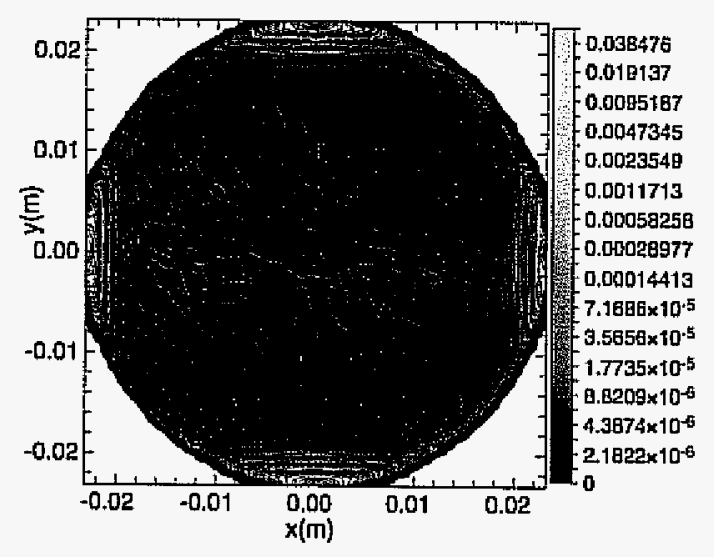

Figure 10 


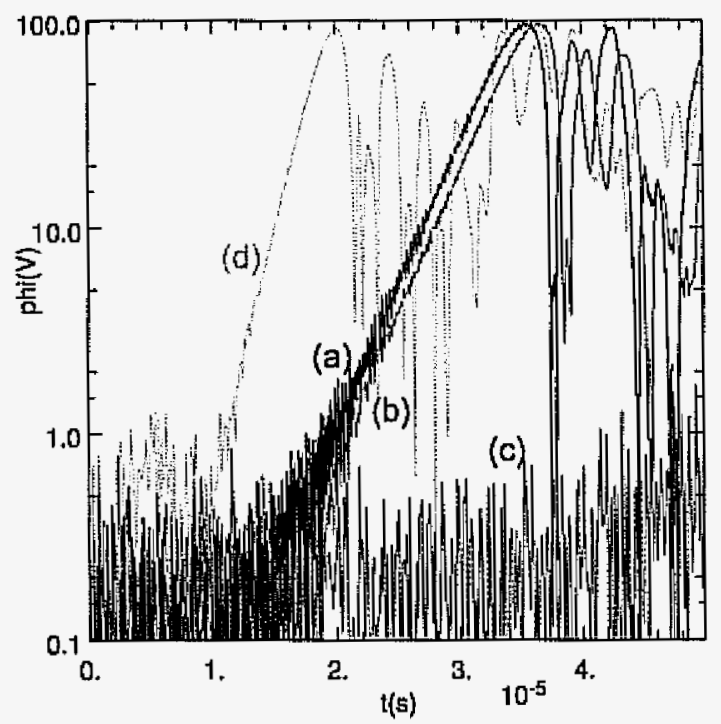

Figure 11 

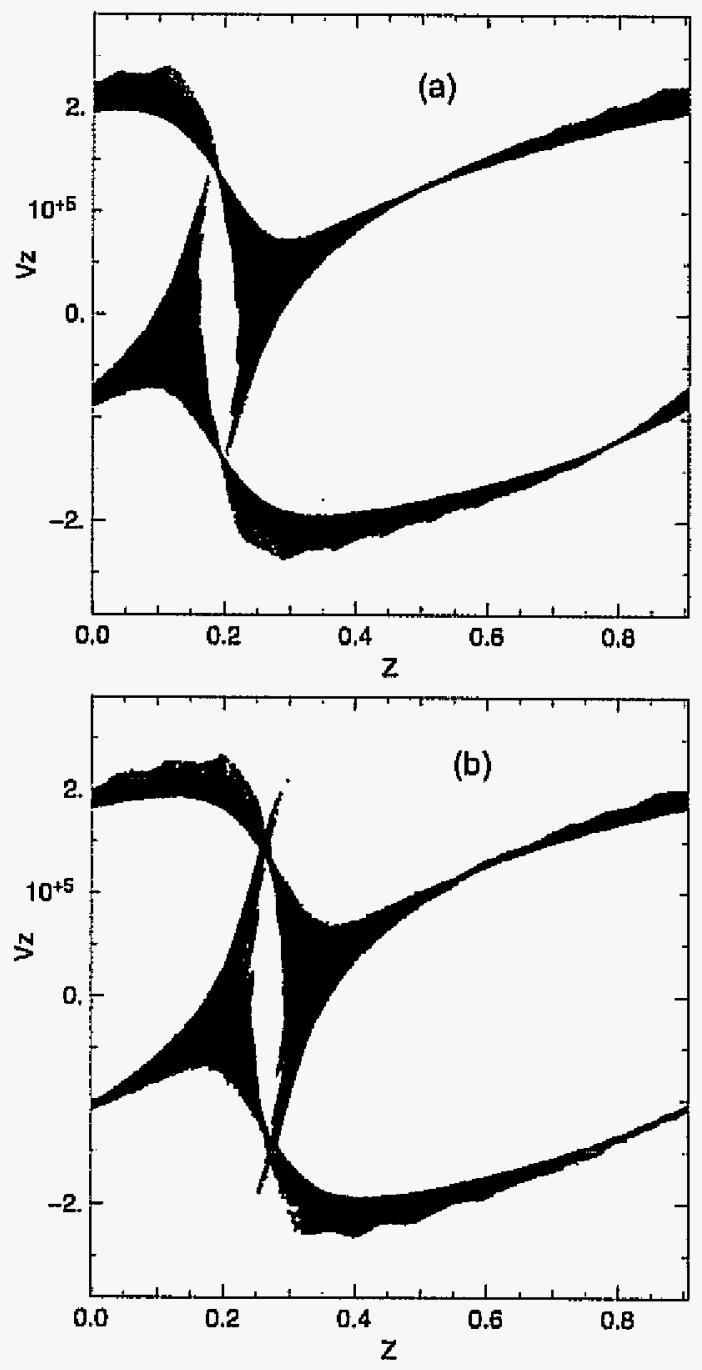

Figure 12 